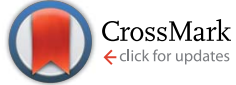

Cite this: RSC Adv., 2015, 5, 19074

Received 29th December 2014 Accepted 9th February 2015

DOI: $10.1039 / c 4 r a 17230 b$

www.rsc.org/advances

\section{High yield synthesis of electrolyte heating assisted electrochemically exfoliated graphene for electromagnetic interference shielding applications $\uparrow$}

\author{
Prashant Tripathi, ${ }^{a}$ Ch. Ravi Prakash Patel, ${ }^{a}$ Abhishek Dixit, ${ }^{a}$ Avanish Pratap Singh, \\ Pawan Kumar, ${ }^{b}$ M. A. Shaz, ${ }^{a}$ Ritu Srivastava, ${ }^{b}$ Govind Gupta, ${ }^{\text {b }}$ S. K. Dhawan, ${ }^{b}$ \\ Bipin Kumar Guptab and O. N. Srivastava*a
}

\begin{abstract}
Herein, we demonstrate a facile one pot synthesis of graphene nanosheets by electrochemical exfoliation of graphite. In the present study, we report a significant increase in the yield of graphene by electrolyte heating assisted electrochemical exfoliation method. The obtained results of heating assisted electrochemically exfoliated graphene (utilizing $\mathrm{H}_{2} \mathrm{SO}_{4}+\mathrm{KOH}+\mathrm{DW}$ ) synthesis clearly exhibit that the yield increases $\sim 4.5$ times i.e. from $\sim 17 \%$ (room temperature) to $\sim 77 \%$ (at $80{ }^{\circ} \mathrm{C}$ ). A plausible mechanism for the enhanced yield based on lattice expansion and vibration of intercalated ions has been put forward and discussed in details. The quality of graphene was examined by Raman, XPS, FTIR, AFM, SEM, TEM/HRTEM and TGA techniques. The Raman as well as morphogenesis results confirm the quality of the graphene nanosheets. We have used this graphene as electromagnetic interference shielding material where a comparatively large quantity of graphene is required. This graphene exhibits enhanced shielding effectiveness ( $46 \mathrm{~dB}$ at $1 \mathrm{~mm}$ thickness of stacked graphene sheets in frequency region 12.4 to $18 \mathrm{GHz}$ ) as compared to conventional electromagnetic interference shielding materials, which is greater than the recommended limit ( $30 \mathrm{~dB}$ ) for techno-commercial applications. Thus the present work is suggestive for future studies on enhancement of yield of high quality graphene by proposed method and the use of synthesized graphene in electromagnetic interference shielding and other possible applications.
\end{abstract}

\section{Introduction}

In modern society, the use of several electronic gadgets, home appliances, commercial as well as industrial equipment and many other luxury electronic devices has produced electromagnetic radiation pollution. ${ }^{1,2}$ The deleterious effect of electromagnetic interference (EMI) on the electronic functionality and human health has initiated the search for suitable absorbing materials to avoid such effects. The EM wave absorbing materials are highly desirable to have a strong absorption over a wide range of frequency and should be lightweight, portable, flexible, corrosion resistant, cost effective and easy to process. A variety of exotic carbon nanomaterials including carbon nanotubes, graphene oxides as well as transition metal oxides and their composites have been studied in the recent years to address this issue., ${ }^{3,4}$ There are number of

${ }^{a}$ Nanoscience Centre, Department of Physics (Centre of Advanced Studies), Banaras Hindu University, Varanasi-221005, India

${ }^{b}$ CSIR-National Physical Laboratory, New Delhi-110 012, India.E-mail: heponsphy@ gmail.com

$\dagger$ Electronic supplementary information (ESI) available. See DOI: $10.1039 / \mathrm{c} 4 \mathrm{ra} 17230 \mathrm{~b}$ important factors like conducting, grounding, electrostatic discharge (ESD) etc. which play key role to the overall performance of the shielding materials. The considerations of many properties e.g. light weight, corrosion free, cost effective, encourage to replaced metals (containing high gravimetric mass) by a variety of new compounds and composite materials (associated with low gravimetric mass with high packing density). Recently, the rapid development of wireless telecommunication, local area networks, radar and many other communication systems forces the huge demand of high performance electromagnetic interference shielding materials. A large number of studies have been devoted to explore highly efficient materials with better microwave absorption with in a wide range of frequency. The realistic approach to address such issues and their legitimate solution for EMI problem is to search a material which exhibits moderate electrical conductivity and high dielectric loss. ${ }^{5,6}$ Therefore, in past, a range of materials including very thin sheets of metals, different forms of carbon, ${ }^{7,8}$ e.g., single or multi-walled carbon nanotubes (CNTs), ${ }^{\mathbf{4}}$ carbon fibers, ${ }^{\mathbf{9}, 10}$ graphite and its new exotic forms ${ }^{\mathbf{1 1 , 1 2}}$ such as expanded graphite, ${ }^{\mathbf{1 3}}$ reduced graphene oxide (rGO), ${ }^{\mathbf{1 4}}$ carbon black ${ }^{15}$ graphene,${ }^{12}$ conducting polymers, dielectric, 
magnetic materials and their composites have been used for EMI shielding in automobile applications.

At present, graphene is the main hub for scientific and industrial community due to their potential applications in many areas, including EMI shielding. Even, graphene composites are much superior to the well-established CNTs composites for EMI shielding. This is because graphene based composites have better thermal stability, more stiff, low cost (compared to CNTs) and have capability of forming a thin film, paper or coating for EMI shielding and electrostatic charge dissipation (ESD) properties. The ultra-high thermal conductivity and lower density (four times as compared to copper) make graphene preferable for packing microelectronic device in the form of composite thin film, paper or coating. Our previous efforts based on graphene encourage us to search more and more new kind of composites with graphene. ${ }^{\mathbf{1 , 1 4}}$ The present investigation is one of the innovative efforts to synthesize graphene on the large scale for EMI shielding, which is highly desirable for practical applications. Most of the synthesis methods have limited their scope due to lack of high yield. At present, a variety of techniques for the synthesis of graphene have been developed. These can be broadly categorized as mechanical, physical and chemical methods. Out of these, chemical methods are best suited for producing graphene of good quality with high yield and low production cost. ${ }^{16}$ Among all methods, the production of graphene through electrochemical exfoliation of graphite seems to score over the thermal exfoliation of graphite oxide. The main drawback of thermal exfoliation invariably leads to creation of graphene containing several oxygen functionalities. Attempts to remove oxygen functionalities have been made through chemical treatments e.g. processing through hydrazine, $\mathrm{NaBH}_{4}$ etc. ${ }^{18,19}$ However, this is a tedious job and it involves toxic process as well as introduces impurities and defects in the resulting graphene. ${ }^{\mathbf{1 7}, 18}$ Production of graphene sheets (GS) directly by electrochemical exfoliation in ionic or acidic electrolytes bypasses the above said difficulties. It has been found that electrochemical exfoliation may lead to optimum alternative for large area, good quality graphene with high yield which is required for present application.

Herein, we report the large scale synthesis of graphene through electrochemical exfoliation method and explore the detailed studies about the method and their effect of electrolyte heating on the yield as well as quality of graphene. Although many reports has been published on the exfoliation of graphene nanosheets such as ball milling, ${ }^{19}$ chemical exfoliation ${ }^{20}$ and electrochemical synthesis. ${ }^{21}$ We had earlier noticed that electrolyte heating significantly affects the electrochemical process. ${ }^{22}$ Addressing on the above problem, we attempted to investigate the effect of increasing temperature of electrolyte on the yield and quality of graphene. It has been found that the electrolyte heating does indeed affect the yield and quality of graphene. For example as against yield of $\sim 17 \%$ for graphite/ $\mathrm{H}_{2} \mathrm{SO}_{4}$-diluted with $\mathrm{KOH} / \mathrm{Pt}$, with electrolyte at room temperature, the corresponding yield for heating assisted electrolyte at $80{ }^{\circ} \mathrm{C}$ is $\sim 77 \%$. To the best of our knowledge, effect of electrolyte heating on graphene higher yield has not been studied till date.
Also graphene yield of $\sim 77 \%$ is possibly the highest yield reported so far for the synthesis through electrochemical exfoliation. Therefore, the present work may be one step ahead towards the synthesis of graphene with high yield which is highly required for such applications where large quantities of graphene required for e.g. EMI shielding application. Furthermore, the quality of as-synthesized graphene in present investigation has been examined by several techniques such as Raman, XPS, FTIR, AFM, SEM, TEM/HRTEM and TGA. Moreover, the shielding effectiveness strongly depends on the thickness of the sheets. The tunability of EMI shielding through thickness variation of the graphene has been evaluated for desired value of shielding effectiveness.

\section{Experimental}

\subsection{Electrochemical exfoliation process for graphene synthesis}

Electrochemical exfoliation of graphite was carried out in an electrolytic cell. Graphite foil (Alfa Aesar) of size $10 \mathrm{~mm} \times 10$ $\mathrm{mm} \times 1 \mathrm{~mm}$ was used as source of graphene (working electrode) for electrochemical exfoliation. The graphite foil was attached to a tungsten wire (diameter $\sim 0.1 \mathrm{~mm}$ ) through silver paste. To protect contacts, it was covered by rapid repair glue used in dentistry. A Pt wire (0.3 mm diameter, $3 \mathrm{~cm}$ long) was employed as counter electrode and placed parallel to the working electrode with a separation of $3 \mathrm{~cm}$. Both the electrodes were immersed into the electrolyte and then external DC biases of $+10 \mathrm{~V}$ (which can be ramped to $-10 \mathrm{~V}$ ) were impressed on the graphite electrode. After the graphite exfoliation was accomplished, the product was collected with a polytetrafluoroethylene (PTFE) membrane filter with $0.2 \mu \mathrm{m}$ pore size. For the preparation of graphene sheet suspension they were first repeatedly washed with distilled water (DW) by vacuum filtration. The sheets were then dried and suspended in dimethylformamide (DMF; Qualigens) and subjected to sonication for $20 \mathrm{~min}$. In order to get rid of graphite particles and exfoliated thin graphitic sheets, the suspension was subjected to centrifugation ( $\sim 4000 \mathrm{rpm}$ for $30 \mathrm{~min}$ ). The supernatant after centrifugation was used for further characterizations.

\subsection{Characterizations of electrochemically exfoliated graphene}

The Raman spectra of the exfoliated graphitic sheets were recorded with Horiba Jobin Yuon (HR800) spectrometer model number $\mathrm{H} 45517$ with an argon-ion laser at an excitation wavelength of $514.5 \mathrm{~nm}$. The XPS analysis of the sample was carried out in an ultrahigh vacuum (UHV) chamber equipped with a hemispherical electron energy analyzer (Perkin Elmer, PHI1257) using a non-monochromatic AlK $\alpha$ source (1486.6 eV) with a base pressure of $4 \times 10^{-10}$ Torr at room temperature. Fourier transform infrared spectroscopy (FTIR) was obtained by using a Perkin Elmer FTIR spectrometer (spectrum 100) in transmission mode in the wave number range $500-4000 \mathrm{~cm}^{-1}$. The spectroscopic grade $\mathrm{KBr}$ pellets were used for collecting the spectra. The atomic force microscopy (AFM) images were taken 
with NT-MDT (SOLVER NEXT). The morphology of the graphene sheets were observed by scanning electron microscopy (SEM) (QUANTA-200). Transmission electron microscopy (TEM) measurements were performed employing a FEI Technai-20G ${ }^{2}$ microscope (acceleration voltage $=200 \mathrm{kV}$ ). The resistance of graphene block was measured with a four-point probe system (Keithly 2000 Multimeter). The thermal stability of graphene was characterized by thermogravimetric analysis (Perkin Elmer, Pyris 1 TGA). The measurement was conducted with heating rate of $5{ }^{\circ} \mathrm{C} \min ^{-1}$ from 50 to $800{ }^{\circ} \mathrm{C}$ under nitrogen flow.

\subsection{Preparation of samples for EMI shielding application}

The graphene obtained by electrochemical exfoliation method was sonicated in water for 1 hour. After that it was collected on filter paper (retention: $2.5 \mu \mathrm{m}$ ) by vacuum filtration and the resultant graphene collected on filter paper was dried in oven at $100{ }^{\circ} \mathrm{C}$. Upon drying, the graphene blocks consisting of stacked graphene were cut from filter paper in a rectangular shape with dimensions $22.8 \times 10 \mathrm{~mm}^{2}$ and having thickness of $0.25 \mathrm{~mm}$ and inserted into a copper sample holder connected between the waveguide flanges of a network analyzer. For investigating the effect of thickness of graphene sheets on EMI shielding, various blocks of thicknesses $0.5 \mathrm{~mm}$ (by stacking two graphene blocks obtained above), $0.75 \mathrm{~mm}$ (by stacking three graphene blocks) and $1 \mathrm{~mm}$ (by stacking four blocks) were used. Electromagnetic shielding measurements were recorded by using Agilent E8362B Vector Network Analyzer in the $12.4-18 \mathrm{GHz}$ microwave range.

\section{Results and discussion}

\subsection{Enhanced yield of graphene}

As outlined earlier a part of the work describe in this communication is on enhancing yield of graphene synthesis through electrochemical exfoliation. Therefore the experimental process involved for this synthesis will now be given. We have first prepared 2.0 $\mathrm{M}$ and 0.2 $\mathrm{M} \mathrm{H}_{2} \mathrm{SO}_{4}$ (Fisher Scientific, AR grade; $98 \%$ ) as acidic electrolyte. In order to reduce the acidity of the electrolyte, we have prepared another electrolyte with $0.25 \mathrm{M}$ $\mathrm{H}_{2} \mathrm{SO}_{4}$ mixed with $11 \mathrm{ml} \mathrm{KOH}$ \{Fisher Scientific\}, (30\% solution). Salt $\mathrm{KOH}$ is added to lower the acidity of $\mathrm{H}_{2} \mathrm{SO}_{4}$. This will partially avoid the oxidation of graphene resulting from the electrochemical exfoliation. The above said electrolyte has been found optimum electrolyte for preparation of graphene sheets through electrochemical exfoliation.

In order to find out the effect of heating the electrolyte on the yield of graphene, we heated the electrolyte on a hot plate. After the electrolyte has achieved steady temperature, the voltage was impressed between the electrodes. When voltage of $10 \mathrm{~V}$ was applied to the graphite electrode, the graphite foil began to dissociate into the thin sheets and spread into the electrolyte solution. The sheets obtained after exfoliation of graphite were washed repeatedly with distilled water by vacuum filtration. The sheets were then dried and suspended in water and subjected to sonication for $20 \mathrm{~min}$, finally forming a black suspension. After that, the suspension was centrifuged at $4000 \mathrm{rpm}$ for $30 \mathrm{~min}^{{ }^{19}}$
Table 1 Effect of electrolyte heating on yield of graphene

Percentage yield of graphene (wt\%) at different temperatures

\begin{tabular}{|c|c|c|c|c|}
\hline Electrolyte & RT (300 K) & $\begin{array}{l}40{ }^{\circ} \mathrm{C} \\
(313 \mathrm{~K})\end{array}$ & $\begin{array}{l}60{ }^{\circ} \mathrm{C} \\
(333 \mathrm{~K})\end{array}$ & $\begin{array}{l}80^{\circ} \mathrm{C} \\
(353 \mathrm{~K})\end{array}$ \\
\hline $2.0 \mathrm{M} \mathrm{H}_{2} \mathrm{SO}_{4}$ & $\sim 6$ & $\sim 6.30$ & $\sim 7$ & $\sim 8$ \\
\hline $0.2 \mathrm{M} \mathrm{H}_{2} \mathrm{SO}_{4}$ & $\sim 7$ & $\sim 8$ & $\sim 12$ & $\sim 13$ \\
\hline $\mathrm{H}_{2} \mathrm{SO}_{4}+\mathrm{KOH}+\mathrm{DW}$ & $\sim 17$ & $\sim 28$ & $\sim 44$ & $\sim 77$ \\
\hline $\mathrm{H}_{2} \mathrm{PO}_{4}+\mathrm{KOH}+\mathrm{DW}$ & $\sim 12$ & $\sim 13$ & $\sim 19$ & $\sim 35$ \\
\hline $\mathrm{HClO}_{4}+\mathrm{NaOH}+\mathrm{DW}$ & $\sim 13$ & $\sim 17$ & $\sim 24$ & $\sim 43$ \\
\hline
\end{tabular}

The supernatant after centrifugation was consisted of few layers of graphene and the precipitated samples were relatively thick graphite flakes. The yield of graphene sheets was calculated by estimating the fractions of graphene sheets and graphite were based on the mass measurement of the dispersed sheets in supernatant (graphene) and precipitated part of solid at bottom (graphite) after centrifugation of exploited solution which is well established and also reported in literature. ${ }^{23}$ For the better reproducibility, the yield of graphene was estimated after collection of 20 to 25 electrochemical statistical runs under identical conditions. The electrolyte was heated upto three different temperatures namely $40{ }^{\circ} \mathrm{C}, 60^{\circ} \mathrm{C}$ and $80^{\circ} \mathrm{C}$. Since the electrolyte contains water so temperature beyond $100{ }^{\circ} \mathrm{C}$ is not feasible. The yield of graphene was obtained by using different electrolytes, which is shown in Table 1 . The yield of graphene nanosheets have increased upto $\sim 13 \%$ at $80{ }^{\circ} \mathrm{C}$ when $0.2 \mathrm{M}$ $\mathrm{H}_{2} \mathrm{SO}_{4}$ as an electrolyte as compared to the yield of $\sim 7 \%$ at room temperature. The graphene yield for electrolyte with $\mathrm{H}_{2} \mathrm{SO}_{4}$ diluted with DW and $\mathrm{KOH}$ (composition $2.4 \mathrm{~g}$ of $\mathrm{H}_{2} \mathrm{SO}_{4}$ and 11 $\mathrm{ml}$ of $30 \% \mathrm{KOH}$ in $100 \mathrm{ml} \mathrm{DW}$ ) was $\sim 77 \%$ at $80{ }^{\circ} \mathrm{C}$ as compared to $\sim 17 \%$ at room temperature. It may be pointed out that this yield is very high. Fig. S1 (see in ESI $\dagger$ ) shows the optical images of the resulting thin sheets after exfoliation at RT and $80{ }^{\circ} \mathrm{C}$. The electrolyte heating, therefore, provides a convenient way for increasing the yield of graphene. To examine the generality of the electrolyte heating effect, two other electrolytes $0.25 \mathrm{M}$ $\mathrm{H}_{3} \mathrm{PO}_{4}$ (Qualigens, 88\%) diluted with $11 \mathrm{ml}$ of $\mathrm{KOH}(30 \%)$ and $0.25 \mathrm{M} \mathrm{HClO}_{4}$ (Qualigens, 60\%) diluted with $11 \mathrm{ml} \mathrm{NaOH}$ \{Fisher Scientific\} $(30 \%)$ were taken. The yield of graphene at room temperature and $40{ }^{\circ} \mathrm{C}, 60^{\circ} \mathrm{C}, 80^{\circ} \mathrm{C}$ were evaluated for all the three electrolytes. For $\mathrm{H}_{3} \mathrm{PO}_{4}(\mathrm{KOH})$ and $\mathrm{HClO}_{4}(\mathrm{NaOH})$ the increase in the yield was from $\sim 12 \%$ (RT) to $\sim 35 \%\left(80{ }^{\circ} \mathrm{C}\right)$ and from $\sim 13 \%$ (RT) to $\sim 43 \%\left(80{ }^{\circ} \mathrm{C}\right)$ respectively.

The different extents of increase in yield of graphene for different acidic electrolytes are presumably due to different sizes of the intercalating ions and also the details of the nature of interaction of intercalating ions with graphitic layers. The schematic representation of electrochemical exfoliation of graphene as well as its proposed application (to be described in next section) is shown in Fig. 1.

Fig. 2 exhibits a representative Raman spectrum (excited by $514 \mathrm{~nm}$ ) for the exfoliated graphitic thin sheets. For comparison a typical Raman spectra from starting graphite material is 


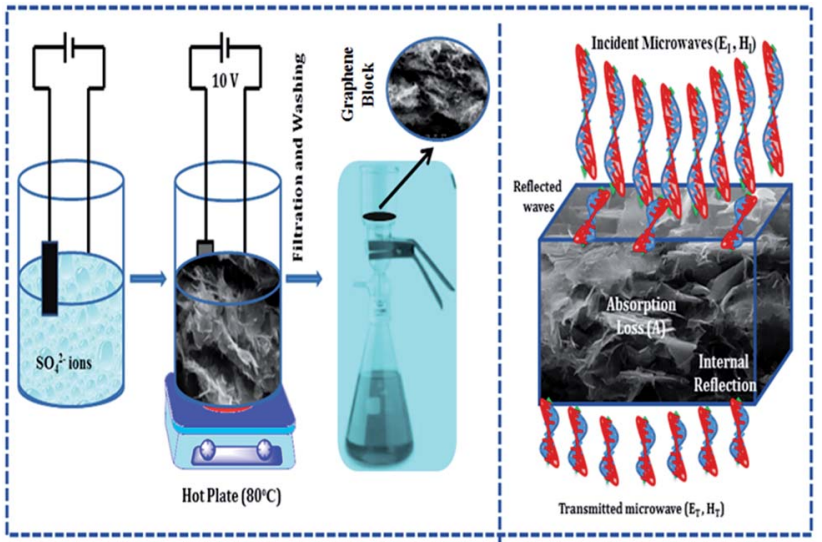

Fig. 1 Schematic of electrochemical exfoliation of graphene and its application in EMI shielding

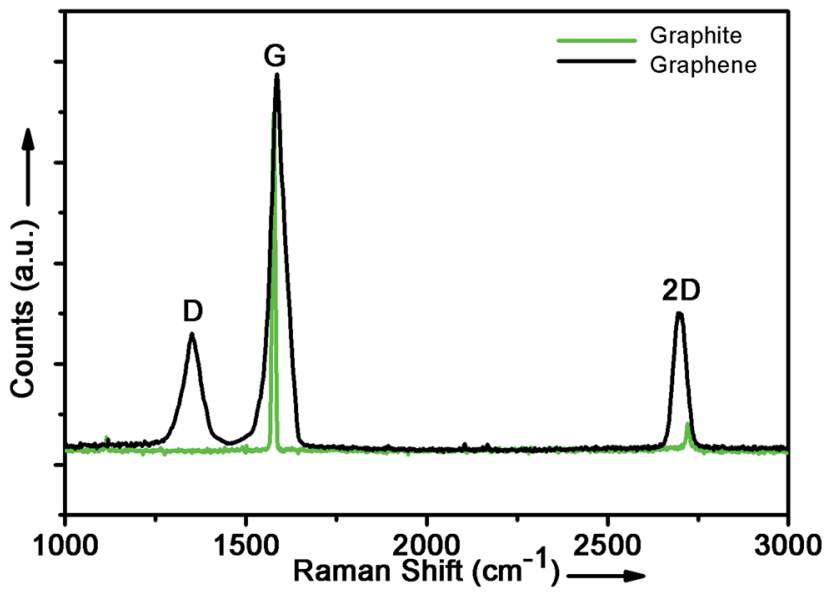

Fig. 2 Raman spectra of starting material (graphite) and the product after electrochemical exfoliation.

superimposed. A strong G-band at $1575 \mathrm{~cm}^{-1}$ can be easily seen in Fig. 2. After exfoliation the graphitic sheets shows a strong Gband at $1584 \mathrm{~cm}^{-1}$. However, a D-band at $1351 \mathrm{~cm}^{-1}$ also appears, the $\mathrm{D}$-band is representative of disorder. This is absent in the spectra of starting graphite. The $I_{\mathrm{D}} / I_{\mathrm{G}}$ ratio in exfoliated graphitic sheets is 0.33 . This is suggestive of the partial oxidation on exfoliation of graphene..$^{24,25}$ The presence of $2 \mathrm{D}$ peak around $2698 \mathrm{~cm}^{-1}$ is clear indication that these thin graphitic sheets are graphene. The $I_{2 \mathrm{D}} / I_{\mathrm{G}}$ ratio in exfoliated graphitic sheets is 0.39 . The obtained Raman result clearly demonstrates the quality of graphene nanosheets.

Futhermore, for elemental composition study of exfoliated graphene, XPS had been performed. The survey scan of exfoliated graphene demonstrated in Fig. 3(a). The XPS core level spectra of C1s and O1s region for exfoliated graphene are shown in Fig. 3(b) and (c). Fig. 3(d) reveals the deconvoluted spectra of $\mathrm{C} 1 \mathrm{~s}$ where the presence of $\mathrm{C}-\mathrm{C}, \mathrm{C}=\mathrm{C}$ and $\mathrm{C}-\mathrm{O}$ which confirms the quality of exfoliated graphene, can be clearly seen.

Fig. 4(a) and (b) exhibits 2D and 3D AFM images of the exfoliated graphene sheets. The sample for AFM was prepared
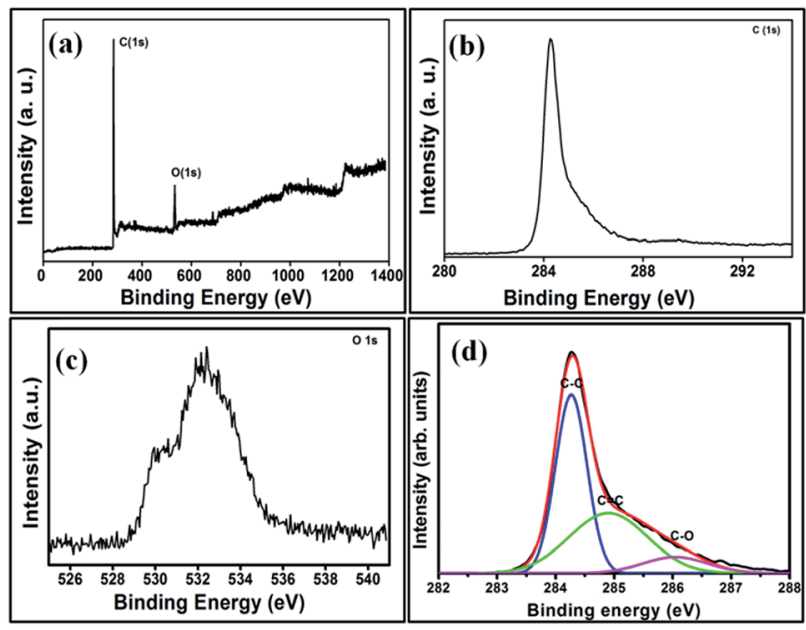

Fig. 3 (a) XPS survey scan of exfoliated graphene (b) and (c) core-level spectra of $\mathrm{C} 1 \mathrm{~s}$ and $\mathrm{O} 1 \mathrm{~s}$, respectively and (d) deconvoluted XPS spectrum of $\mathrm{C} 1 \mathrm{~s}$ core level of exfoliated graphene.

by drop casting solution of graphene dispersed in $\mathrm{DMF}$ on $\mathrm{SiO}_{2}$ substrate. The high resolution 2D and 3D AFM images of the exfoliated graphene sheets are shown in Fig. 4(c) and (d). The smallest thickness of the graphene sheets are $\sim 2.4 \mathrm{~nm}$ as shown in Fig. S2 (see in ESI $\dagger$ ). Some oxygen groups are expected to be present at the graphene surface. The thickness of the exfoliated graphene layers will be larger than the ideal thickness of $0.65 \mathrm{~nm}$ on $\mathrm{SiO}_{2}$ for monolayer, ${ }^{26}$ here it is $\sim 0.80 \mathrm{~nm}$. Thus the thickness of $\sim 2.4 \mathrm{~nm}$ can be taken to represent three layers of graphene. The statistics histogram for the thickness demonstrated in Fig. S3 (see ESI†). The electrochemically exfoliated graphene layers in the present case thus can be taken to correspond to three/few layers of graphene.

The surface morphology and microstructure analysis of exfoliated graphene have been examined by using SEM and TEM techniques. Fig. 5(a) represents the cross sectional SEM
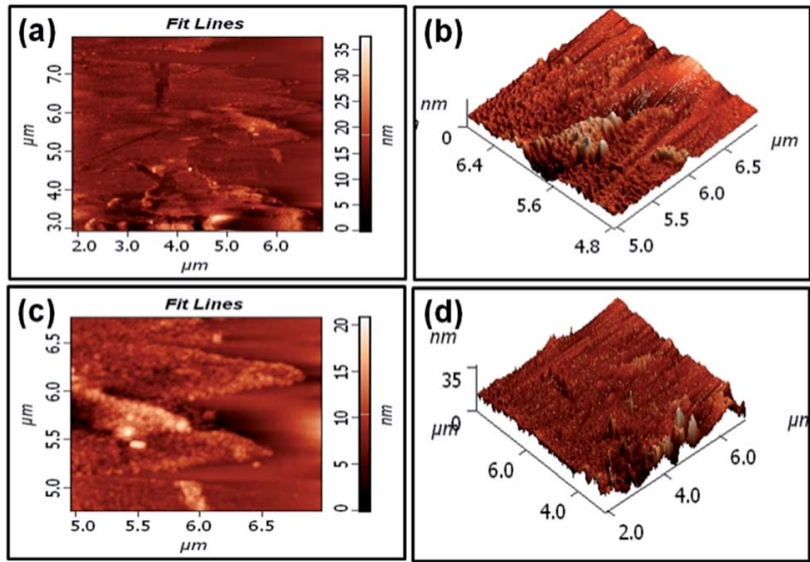

Fig. 4 (a) and (b) Typical 2D and 3D AFM images of exfoliated graphene sheets deposited on $\mathrm{SiO}_{2} / \mathrm{Si}$ substrate respectively,(c) and (d) high-resolution typical 2D and 3D AFM images for exfoliated graphene sheets deposited on $\mathrm{SiO}_{2} / \mathrm{Si}$ substrate respectively. 

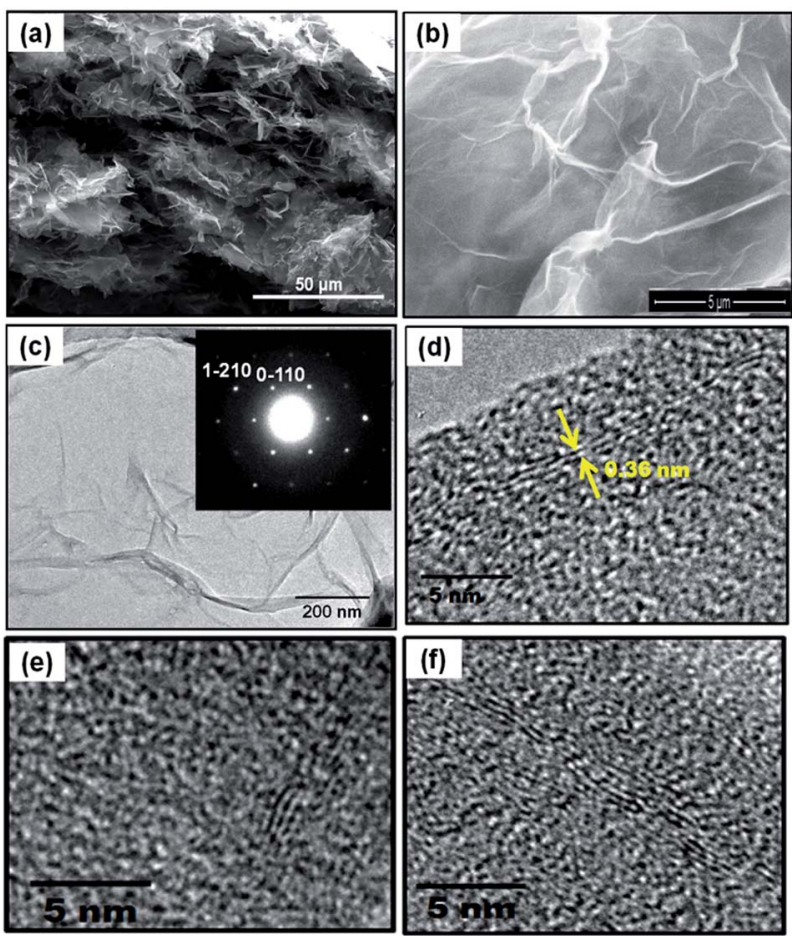

Fig. 5 (a) shows the cross-sectional SEM image of stacked graphene block. (b) SEM image of as-synthesized electrochemically exfoliated graphene sheet. (c) Typical TEM micrograph of exfoliated graphene sheet and inset shows SAED pattern of exfoliated graphene and (d), (e) and (f) exhibit the HRTEM micrographs of exfoliated graphene.

image of stacked block of graphene sheets which is further used for EMI shielding measurements. In order to ensure the quality and surface morphology of the graphene, we have also performed SEM microscopy of as-synthesized electrochemically exfoliated graphene sheets as shown in Fig. 5(b). The SEM micrograph brings out the morphology of the exfoliated graphene sheets. It can be easily seen that the thin graphene regions together with ruptures/cracks produced due to electrochemical exfoliation process. Fig. 5(c) exhibits a typical TEM micrograph of exfoliated graphene supported on a holey carbon grid. The inset of Fig. 5(c) exhibits SAED pattern of exfoliated graphene. The six fold diffraction pattern bringing out the graphitic nature of the exfoliated thin sheets. To identify the number of graphene layers, efforts were made to take HRTEM images of exfoliated graphene. The Fig. 5(d)-(f) reveals the HRTEM images of exfoliated graphene. Fig. 5(d) demonstrated that the estimated $d$-spacing of exfoliated graphene is $\sim 0.36 \mathrm{~nm}$ which is comparable to graphene. The HRTEM images $\{$ Fig. 5(d)-(f)\} reveals that the layer number of these exfoliated graphene ranges from two to four. Thus the electrochemical exfoliation process adopted in the present investigation produces few layers of graphene.

Fig.6(a) exhibits the room temperature $I-V$ characteristics of graphene block (thickness $\sim 0.25 \mathrm{~mm}$ ). The calculated surface resistance of graphene block (thickness $\sim 0.25 \mathrm{~mm}$ ) is $\sim 2.14 \mathrm{k} \Omega$ $\mathrm{m}^{-2}$. To examine the thermal stability of graphene block, the thermogravimetric analysis (TGA) has been performed. The

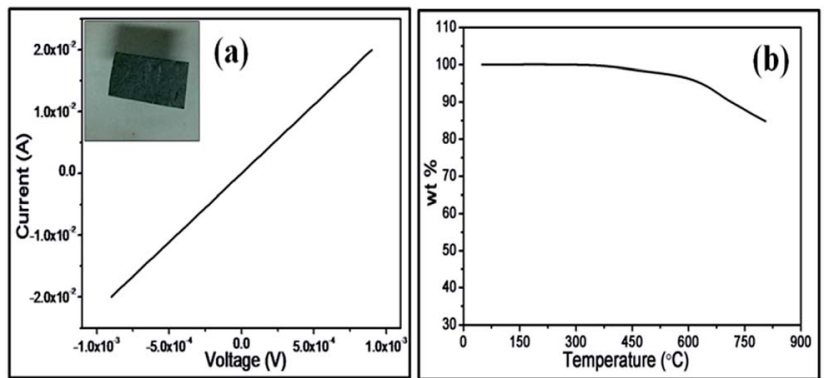

Fig. 6 (a) $I-V$ characteristic of graphene block and inset shows the optical image of graphene block. (b) The TGA curve of exfoliated graphene.

TGA curve of graphene block is illustrated in Fig. 6(b). The TGA curve demonstrates that graphene block is thermally stable in nitrogen ambient until $\sim 635{ }^{\circ} \mathrm{C}$, above which a continuous weight loss occurs. The weight loss at high temperature of graphene block is due the oxidation of the carbon atoms into $\mathrm{CO}_{2}$. The FTIR spectrum of graphene block is shown in Fig. S4 (see ESI†). The FTIR spectrum of graphene block exhibits the presence of $\mathrm{C}-\mathrm{O}-\mathrm{C}, \mathrm{C}-\mathrm{H}, \mathrm{C}=\mathrm{C}$ and $\mathrm{C}-\mathrm{O}-\mathrm{H}$.

\subsection{Feasible mechanism for enhanced yield of graphene}

It is now generally believe that the electrochemical exfoliation producing graphene takes place due to intercalation of ions (i.e. $\mathrm{SO}_{4}{ }^{2-}$ ) of the electrolyte. Water used to dilute the acid is always present. For acidic electrolytes the oxidation takes place at the graphitic sheets plane edges or grain boundaries. This is believed to open up to successive graphitic layers. This facilitates intercalation of $\mathrm{SO}_{4}{ }^{2-}$ ion (size $\sim 4.60 \AA$ ) in the layers which dilates the interlayer spacing of graphitic planes (3.35 $\mathrm{A})$. The $\mathrm{SO}_{4}{ }^{2-}$ eventually gets dissociated to $\mathrm{SO}_{2}$. The van der Waals bond between the layers gets considerably weakened. This results in tearing of graphite layer(s) producing very thin graphitic sheets (graphene layers). For the electrolyte containing $\mathrm{KOH}$ besides $\mathrm{H}_{2} \mathrm{SO}_{4}$, the $\mathrm{OH}^{-}$ions in addition to $\mathrm{SO}_{4}{ }^{2-}$ ions will also be present. Therefore, the $\mathrm{OH}^{-}$ions (size $\sim 2.50 \AA$ A) will

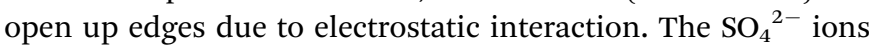
will then find it easier to get intercalated in the graphitic layers.

As regards the effect of electrolyte heating on the yield of graphene is related, the following two effects are relevant-

(1) The increase of graphitic interlayer spacing as a result of electrolyte heating.

(2) The effect of thermal vibrations of the intercalating ions due to electrolyte heating.

The graphite electrode of the electrochemical cell is immersed in the electrolyte. Thus when the electrolyte is heated (up to $80{ }^{\circ} \mathrm{C}$ ) and steady state is achieved, the graphite will undergo thermal expansion. The expansion in the direction perpendicular to the graphite basal layers (i.e. along $c$ lattice direction) will be relevant. This is so since the graphene is produced due to intercalation of ions of the electrolyte in the successive graphitic layers. It is known that at room temperature the lattice parameters of graphite are $a=2.456 \AA$, and $c=$ $6.693 \AA$ A. The 'a' parameter known to exhibit slight decrease up 
to about $400{ }^{\circ} \mathrm{C}$, on the other hand thermal expansion along the $c$-direction is large. ${ }^{27}$ The thermal expansion coefficient at $50{ }^{\circ} \mathrm{C}$ is $>0.96 \times 10^{-6}$. The expression for the expansion of interlayer spacing has been found to be $d=3.3525+90.54 \times 10^{-6} T+(6.33$ $\left.\times 10^{-9}\right) T^{2}=3.3525+90.54 \times 10^{-6} \AA^{28}$ Thus for $80{ }^{\circ} \mathrm{C}$, the interlayer spacing will increase from $3.3525(\sim 3.35 \AA)$ to 3.3595 $(\sim 3.36 \AA)$, thus the change in interlayer spacing is small. However, it should be noted that the van der Waals potential will vary approx as $1 / d^{6}$. This will facilitate the intercalation of the ions originating from the electrolyte. Thus, the electrolyte ion $\mathrm{SO}_{4}{ }^{2-}(4.60 \AA)$ from $\mathrm{H}_{2} \mathrm{SO}_{4}$ will find it easier to enter in expanded graphite with larger interlayer spacing (3.36 ̊̊). Efficient intercalation of ions resulting from the above effects will lead to efficient electrochemical exfoliation and hence higher yield of graphene.

The second effect as outlined in the above is in regards to thermal vibration of the ions coming from heating the electrolyte. The thermal vibration which will be isotropic will increase as a result of increase of the temperature of the electrolyte. This enhancement of thermal vibration would get somewhat damped once the ion gets intercalated. The vibration of the ion perpendicular to basal graphitic planes will produce a kind of vertical jerk to successive graphitic layers as they move under the applied electric field. This will eventually facilitate exfoliation efficiency and thus increases the yield of graphene. It may be pointed out that the graphitic layers are bonded by weak van der Waals bond. The interlayer bonding energy of graphite is $\sim 31 \pm 2 \mathrm{meV} .{ }^{29}$ The thermal energy at $80{ }^{\circ} \mathrm{C}$ (the maximum temperature up to which the electrolyte has been heated) corresponds to $\sim 30 \mathrm{meV}$. These estimates suggest qualitatively that the increase in thermal energy of ions coming from heating the electrolyte is expected to weaken/break the van der Waals bond between graphitic layers. This will apparently facilitate electrochemical exfoliation resulting in increase of graphene yield. Based on the present experiments it is difficult to decide which of the two effects, the thermal expansion of the graphite electrode or increased vibration of intercalating ions, will be more effective in increasing the yield of the graphene. Possibly both effects may collectively lead to increase in the yield of graphene.

\subsection{Relevant basics of EMI shielding}

Being highly conducting and high dielectric loss, graphene is an ideal material for EMI shielding to block polluting radiation in electronic gadgets.

The EMI Shielding Effectiveness (SE) of a material is defined as the ratio of transmitted power to the incident power and is given by

$$
\mathrm{SE}(\mathrm{dB})=-10 \log \left(\frac{P_{\mathrm{T}}}{P_{\mathrm{I}}}\right)
$$

where, $P_{\mathrm{T}}$ and $P_{\mathrm{I}}$ are the transmitted and incident electromagnetic powers, respectively. For a shielding material, total $\mathrm{SE}=$ $\mathrm{SE}_{\mathrm{R}}+\mathrm{SE}_{\mathrm{A}}+\mathrm{SE}_{\mathrm{M}}$, where $\mathrm{SE}_{\mathrm{R}}, \mathrm{SE}_{\mathrm{A}}$, and $\mathrm{SE}_{\mathrm{M}}$ are shielding effectiveness due to reflection, absorption, and multiple reflections, respectively.
In two port network, $S$-parameters $S_{11}\left(S_{22}\right), S_{12}\left(S_{21}\right)$ represents the reflection and the transmission coefficients given as

$$
\begin{aligned}
& T=\left|\frac{E_{\mathrm{T}}}{E_{\mathrm{I}}}\right|^{2}=\left|S_{21}\right|^{2}=\left|S_{12}\right|^{2} \\
& R=\left|\frac{E_{\mathrm{R}}}{E_{\mathrm{I}}}\right|^{2}=\left|S_{11}\right|^{2}=\left|S_{22}\right|^{2}
\end{aligned}
$$

And absorption coefficient $(A)=1-R-T$.

Here, it is noted that the absorption coefficient is given with respect to the power of the incident electromagnetic wave. If the effect of multiple reflections between both interfaces of the material is negligible, then the relative intensity of the effectively incident electromagnetic wave inside the material after reflection is based on the quantity $(1-R)$. Therefore, the effective absorbance $\left(A_{\text {eff }}\right)$ can be described as $A_{\text {eff }}=(1-R-T) /$ $(1-R)$ with respect to the power of the effective incident electromagnetic wave inside the shielding material. It is convenient to express the reflectance and effective absorbance in the form of $-10 \log (1-R)$ and $-10 \log \left(1-A_{\text {eff }}\right)$ in decibel $(\mathrm{dB})$, respectively, which give $\mathrm{SE}_{\mathrm{R}}$ and $\mathrm{SE}_{\mathrm{A}}$ as

$$
\mathrm{SE}_{\mathrm{R}}=-10 \log (1-R)
$$

and

$$
\mathrm{SE}_{\mathrm{A}}=-10 \log \left(1-A_{\text {eff }}\right)=-10 \log \left(\frac{T}{1-R}\right)
$$

For the material, the skin depth $(\delta)$ is the distance up to which the intensity of the electromagnetic wave decreases to $1 / e$ of its original strength. The skin depth is related to angular frequency, relative permeability \& total conductivity $\sigma_{\mathrm{T}}=\sigma_{\mathrm{dc}}+\sigma_{\mathrm{ac}}$. According to electromagnetic theory, for electrically thick samples $(t>\delta)$, EMI SE for the plane electromagnetic wave is given ${ }^{30}$ as

$$
\mathrm{SE}(\mathrm{dB})=\mathrm{SE}_{\mathrm{R}}(\mathrm{dB})+\mathrm{SE}_{\mathrm{A}}(\mathrm{dB})
$$

and dependence of SE on dielectric properties \& magnetic properties can be expressed as

$$
\begin{gathered}
\mathrm{SE}_{\mathrm{R}}(\mathrm{dB}) \approx 10 \log \left(\sigma_{\mathrm{ac}} /\left(16 \omega \varepsilon_{0} \mu_{\mathrm{r}}\right)\right) \\
\mathrm{SE}_{\mathrm{A}}(\mathrm{dB})=20\left\{\frac{t}{\delta}\right\} \log e=20 t \sqrt{\frac{\mu_{\mathrm{r}} \omega \sigma_{\mathrm{ac}}}{2}}=8.68\left(\frac{t}{\delta}\right)
\end{gathered}
$$

where $\sigma_{\mathrm{ac}}$ depends on the dielectric properties $\left(\sigma_{\mathrm{ac}}=\omega \varepsilon_{0} \varepsilon^{\prime \prime}\right)$ of the material, $\omega$ is the angular frequency $(\omega=2 \pi f), \varepsilon_{0}$ is the free space permittivity, and $\mu_{\mathrm{r}}$ is the relative magnetic permeability of the sample. In eqn (6), the first term is related to the reflection of the electromagnetic wave and contributes as the SE due to reflection. The second term expresses the loss due to the absorption of the wave when it passes through the shielding material. In microwave range, the contribution of the second part becomes more as compared to the reflection term. Eqn (7) and (8) reveal that with the increase in frequency, the $S E_{R}$ value decreases while the contribution of $\mathrm{SE}_{\mathrm{A}}$ increases. 


\subsection{EMI shielding from stacked graphene blocks}

Fig. 7(a) and (b) show the variation of the SE with frequency in the 12.4-18 GHz range. From the experimental measurement, the $\mathrm{SE}$ due to absorption $\left(\mathrm{SE}_{\mathrm{A}}\right)$ of graphene has been found to vary from 9.14-37.71 dB with increase in the stacking thickness of graphene sheet while the $\mathrm{SE}_{\mathrm{R}}$ remains nearly constant at 7.5 $\mathrm{dB}$. Thus, the total SE achieved for the graphene sheet is 17.23 $\mathrm{dB}(0.25 \mathrm{~mm}), 26.23 \mathrm{~dB}(0.5 \mathrm{~mm}), 32.39 \mathrm{~dB}(0.75 \mathrm{~mm})$ and 46.35 $\mathrm{dB}(1 \mathrm{~mm})$. It is observed in the previous studies that the $\mathrm{SE}_{\mathrm{Abs}}$ and $\mathrm{SE}_{\mathrm{Ref}}$ are related to the stacking thickness of the material. It has been observed that for stacked graphene sheet, $\mathrm{SE}$ is mainly governed by absorption while the $\mathrm{SE}_{\mathrm{R}}$ is constant. It is observed that the thickness of the shield has a great influence on the microwave shielding properties as shown in Fig. 4(b).

For as-synthesized stacked graphene sheets, there are three main reasons for the enhanced microwave attenuation performances of the graphene sheets. First, as mentioned above, the extremely thin and high polarity of the stacked graphene sheets
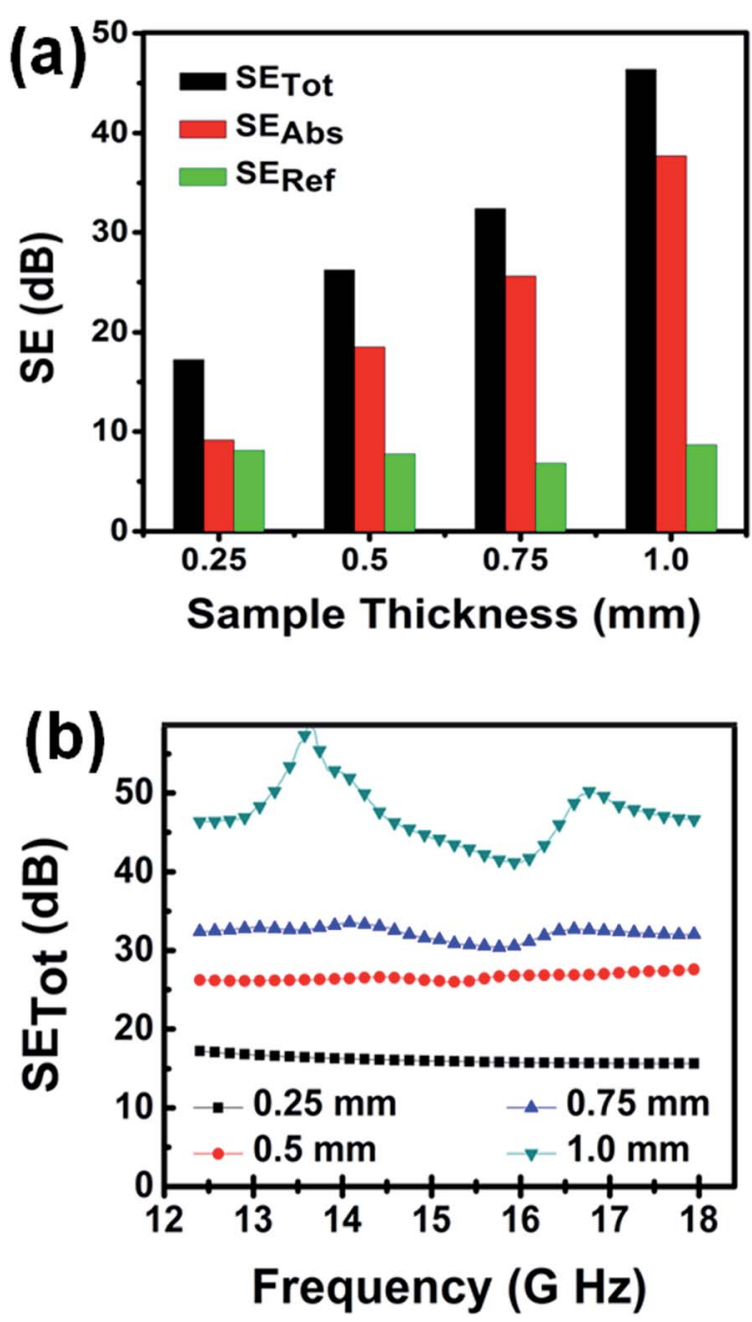

Fig. 7 (a) Variation of shielding effectiveness $\mathrm{SE}_{\mathrm{A}}, \mathrm{SE}_{\mathrm{R}} \& \mathrm{SE}_{\mathrm{T}}$ of graphene blocks with different thickness at fixed frequency; $12.4 \mathrm{GHz}$ and (b) variation of shielding effectiveness $\mathrm{SE}_{\mathrm{A}}, \mathrm{SE}_{\mathrm{R}} \& \mathrm{SE}_{\mathrm{T}}$ with frequency in 12.4-18 GHz for different graphene blocks. may offer more opportunities to induce polarization by EM wave, which improves dielectric losses. ${ }^{1}$ Second, the stacking between graphene sheets increases the conductive paths which leads to enhanced microwave attenuation by converting microwave energy into heat. ${ }^{26}$ Third, extremely thin, flexible and corrugated natures of the graphene sheets increase the propagation paths of the EM wave. The EM wave scattered by corrugated graphene layers, has sufficiently improved attenuation loss with multiple internal reflection loss. When the microwave travels within stacked graphene sheets, the motion of the charge carriers formed oscillatory current ${ }^{31}$ which would consume much EM energy. Thus, the increased scattering coupled with higher conductivity is responsible for the enhanced SE in stacked graphene sheets. Conducting nature of the graphene is beneficial for energy attenuation, while the increasing conductivity would increase the energy conversion effectiveness. Therefore, the results shown in this work indicate the fundamental principles for achieving high value of shielding effectiveness for graphene. According to the reported results in the literature, the defects in graphene may construct localized states near to the Fermi level to increase the radiation attenuation. ${ }^{31,32}$ The microstructure investigations show that the electrochemically synthesized graphene contains clustered defects and residual bonds arising during chemical process. There may oxygenic functional groups (as suggested by FTIR result, ESI Fig. S4†) on the graphene, which might increase the EM wave attenuation performance. Meanwhile, because of the thinnest thickness and high electron mobility; graphene has higher conductivity, which induces strong polarization and loss conductance towards the EM wave. While high gravimetric mass of the graphene makes main contribution to get higher conductivity and higher shielding values simultaneously. Thus, the productions approach as well as results of EMI shielding performance of high quality graphene possesses its legitimate applications in EMI shielding.

\section{Conclusions}

We have successfully demonstrated the large scale synthesis of graphene by electrolyte heating assisted electrochemical method. The obtained results of heating assisted electrochemically exfoliated graphene synthesis clearly exhibits that the yield increases $\sim 4.5$ (from $\sim 17 \%$ to $\sim 77 \%$ ) times, in compared to room temperature synthesis. A plausible mechanism based on the thermal expansion has been put forward and discussed in detailed for higher yield synthesis. The quality of graphene was confirmed by Raman, XPS, FTIR, AFM, SEM, TEM/HRTEM and TGA techniques. The thickness of the stacked graphene sheets has a great influence on the microwave shielding properties. Such high yield good quality graphene exhibits enhanced shielding effectiveness (46 dB at $1 \mathrm{~mm}$ thickness of stacked graphene sheets in frequency region 12.4 to $18 \mathrm{GHz}$ ) compared to conventional electromagnetic interference shielding materials. Thus, this new proposed method could be an ultimate approach to produce high quality graphene in large scale for enhanced microwave shielding application. 


\section{Acknowledgements}

The authors are grateful to DST Nano Science Mission, MNRE, UGC and DRDO India for financial support. We would also like to thanks Dr T. P. Yadav, Prof. A. S. K. Sinha and Prof. P. M. Ajayan for helpful discussions.

\section{Notes and references}

1 A. P. Singh, M. Mishra, P. Sambyal, B. K. Gupta, B. P. Singh, A. Chandra and S. K. Dhawan, J. Mater. Chem. A, 2014, 2, 3581-3593.

2 W.-L. Song, L.-Z. Fan, M.-S. Cao, M.-M. Lu, C.-Y. Wang, J. Wang, T.-T. Chen, Y. Li, Z.-L. Hou, J. Liu and Y.-P. Sun, J. Mater. Chem. C, 2014, 2, 5057-5064.

3 W.-L. Song, M.-S. Cao, M.-M. Lu, S. Bi, C.-Y. Wang, J. Liu, J. Yuan and L.-Z. Fan, Carbon, 2014, 66, 67-76.

4 A. P. Singh, B. K. Gupta, M. Mishra, Govind, A. Chandra, R. B. Mathur and S. K. Dhawan, Carbon, 2013, 56, 86-96.

5 M. Mishra, A. P. Singh, B. P. Singh, V. N. Singh and S. K. Dhawan, J. Mater. Chem. A, 2014, 2, 13159-13168.

6 P. Sambyal, A. P. Singh, M. Verma, M. Farukh, B. P. Singh and S. K. Dhawan, RSC Adv., 2014, 4, 12614-12624.

7 G. Tong, W. Wu, Q. Hua, Y. Miao, J. Guan and H. Qian, J. Alloys Compd., 2011, 509, 451-456.

8 T. GuoXiu, Y. JinHao, M. Ji, Q. M. Yue, G. J. Guo, L. L. Chao, G. Peijun and C. Jianjing, Sci. Sin.: Chim., 2011, 41, 11211126.

9 S. Yang, K. Lozano, A. Lomeli, H. D. Foltz and R. Jones, Composites, Part A, 2005, 36, 691-697.

10 L. Li and D. D. L. Chung, Composites, 1994, 25, 215-224.

11 D. D. L. Chung, Carbon, 2001, 39, 279-285.

12 A. P. Singh, M. Mishra, A. Chandra and S. K. Dhawan, Nanotechnology, 2011, 22, 465701.

13 M. Mishra, A. P. Singh and S. K. Dhawan, J. Alloys Compd., 2013, 557, 244-251.

14 A. P. Singh, P. Garg, F. Alam, K. Singh, R. B. Mathur, R. P. Tandon, A. Chandra and S. K. Dhawan, Carbon, 2012, 50, 3868-3875.
15 N. C. Das, D. Khastgir, T. K. Chakia and A. Chakraborty, Composites, Part A, 2000, 31, 1069-1081.

16 S. Park and R. S. Ruoff, Nat. Nanotechnol., 2009, 4, 217-224. 17 S. Stankovich, D. A. Dikin, R. D. Piner, K. A. Kohlhaas, A. Kleinhammes, Y. Jia, Y. Wu, S. T. Nguyen and R. S. Ruoff, Carbon, 2007, 45, 1558-1565.

18 H. J. Shin, K. K. Sim, A. Benayad, S. M. Yoon, H. K. Park, I. S. Jung, M. H. Jin, H. K. Jeong, J. M. Kim, J. Y. Choi and Y. H. Lee, Adv. Funct. Mater., 2009, 19, 1987-1992.

19 W. Zhao, F. Wu, H. Wu and G. Chen, J. Nanomater., 2010, 15.

20 Y. T. Liu, X. M. Xie and X. Y. Ye, Carbon, 2011, 49, 3529-3537. 21 N. Liu, F. Luo, H. Wu, Y. Liu, C. Zhang and J. Chen, Adv. Funct. Mater., 2008, 18, 1518-1525.

22 A. Chandra, R. N. Pandey, O. N. Srivastava and G. Prasad, Semicond. Sci. Technol., 1991, 6, 137-140.

23 W. Qian, R. Hao, Y. Hou, Y. Tian, C. Shen, H. Gao and X. Liang, Nano Res., 2009, 2, 706-712.

24 C. Mattevi, G. Eda, S. Agnoli, S. Miller, K. A. Mkhoyan, O. Celik, D. Mastrogiovanni, G. Granozzi, E. Garfunkel and M. Chhowalla, Adv. Funct. Mater., 2009, 19, 2577-2583.

25 V. López, R. S. Sundaram, C. Gómez-Navarro, D. Olea, M. Burghard, J. Gómez-Herrero, F. Zamora and K. Kern, Adv. Mater., 2009, 21, 4683-4686.

26 Z. G. Cheng, Q. Y. Zhou, C. X. Wang, Q. A. Li, C. Wang and Y. Fang, Nano Lett., 2011, 11, 767-769.

27 J. B. Nelson and D. P. Riley, Proc. Phys. Soc., London, 1945, 57, 477-486.

28 E. A. Kellett and B. P. Richards, J. Appl. Crystallogr., 1971, 4, 1-8.

29 Z. Liu, J. Z. Liu, Y. Cheng, Z. Li, L. Wang and Q. Zheng, Phys. Rev. B: Condens. Matter Mater. Phys., 2012, 85, 205418.

30 N. F. Colaneri and L. W. Schacklette, IEEE Trans. Instrum. Meas., 1992, 41, 291-297.

31 B. Wen, X. X. Wang, W. Q. Cao, H. L. Shi, M. M. Lu, G. Wang, H. B. Jin, W. Z. Wang, J. Yuan and M. S. Cao, Nanoscale, 2014, 6, 5754-5761.

32 P. C. P. Watts, W. K. Hsu, A. Barnes and B. Chambers, Adv. Mater., 2003, 15, 600-603. 